\title{
A scoping review of crisis teams managing dementia in older people
}

\author{
This article was published in the following Dove Press journal: \\ Clinical Interventions in Aging \\ 3 October 2017 \\ Number of times this article has been viewed
}

\section{Amy Streater ${ }^{1,2}$ \\ Donna Maria \\ Coleston-Shields ${ }^{2}$ \\ Jennifer Yates ${ }^{2}$ \\ Miriam Stanyon ${ }^{2}$ \\ Martin Orrell ${ }^{2}$}

'Research and Development, North East London NHS Foundation

Trust, Ilford, ${ }^{2}$ Institute of Mental Health, University of Nottingham, Nottingham, UK
Correspondence: Amy Streater North East London NHS Foundation Trust, Research and Development, Ist Floor, Maggie Lilley Suite, Goodmayes Hospital, Barley Lane, llford,

Essex IG3 8XJ, UK

Tel +44 300555 I 200 ext 64497

Email amy.streater@nottingham.ac.uk
Background: Research on crisis teams for older adults with dementia is limited. This scoping review aimed to 1) conduct a systematic literature review reporting on the effectiveness of crisis interventions for older people with dementia and 2) conduct a scoping survey with dementia crisis teams mapping services across England to understand operational procedures and identify what is currently occurring in practice.

Methods: For the systematic literature review, included studies were graded using the Critical Appraisal Skills Programme checklist. For the scoping survey, Trusts across England were contacted and relevant services were identified that work with people with dementia experiencing a mental health crisis.

Results: The systematic literature review demonstrated limited evidence in support of crisis teams reducing the rate of hospital admissions, and despite the increase in number of studies, methodological limitations remain. For the scoping review, only half $(51.8 \%)$ of the teams had a care pathway to manage crises and the primary need for referral was behavioral or psychological factors.

Conclusion: Evidence in the literature for the effectiveness of crisis teams for older adults with dementia remains limited. Being mainly cohort designs can make it difficult to evaluate the effectiveness of the intervention. In practice, it appears that the pathway for care managing crisis for people with dementia varies widely across services in England. There was a wide range of names given to the provision of teams managing crisis for people with dementia, which may reflect the differences in the setup and procedures of the service. To provide evidence on crisis intervention teams, a comprehensive protocol is required to deliver a standardized care pathway and measurable intervention as part of a large-scale evaluation of effectiveness.

Keywords: dementia, home treatment, crisis resolution, crisis, mental health, community mental health services

\section{Introduction}

The Dementia UK report ${ }^{1}$ identified people with dementia as significant users of health and social care services. People with dementia occupy a third of beds in acute medical wards, and reducing the stay of people with dementia in hospital by 1 week could generate savings of approximately $£ 80$ million a year. ${ }^{2}$ Yet dementia care is frequently being delivered in an ad hoc and inefficient manner, and consequently older people in the community can experience a "conveyor belt" of care, resulting in residential care, particularly after a crisis incident and subsequent hospital admission. ${ }^{3}$ A crisis can be defined as "a process where there is a stressor(s) that causes an imbalance requiring an immediate decision which leads to a desired outcome, and therefore crisis resolution. If the crisis is not resolved, the cycle continues (pg.2)". ${ }^{4}$ A key failing of service provision is the lack of information and support for people with dementia and 
their carers requiring immediate help..$^{5}$ The Prime Minister's Challenge on Dementia 2020 recognizes the importance of support provided post-diagnosis. ${ }^{6}$ Support for family carers and the provision of rapid, simple interventions or professional support for long-term care home placement can avoid crisis hospital admissions. $^{7}$

Mental health services available for younger people intend to provide coping strategies, attempt to address social and family factors that can lead to a crisis, and encouragement to draw on pre-existing support networks to manage their condition and avoid hospital admissions. ${ }^{8}$ Similar services for older people with dementia are not provisioned as equitably, ${ }^{9}$ and there is a lack of formal evaluation of the provision of these services. ${ }^{10}$ Through joined up preventative and coordinated health care, services can be tailored to enable people with dementia to stay in their own homes, avoiding hospital admissions and crisis situations. ${ }^{11}$ Previous survey research exploring the provision of Crisis Resolution Teams (CRTs) across 79 Trusts in England found that while 99\% of responding Trusts provided acute mental health services, less than a third of Trusts offered the same CRT across age groups through a specialist team, adult CRT, or intermediate care team. ${ }^{10}$ People with dementia were only able to access crisis services in a tenth of areas, with just one in six teams frequently providing services to older people. An exploration of the attitudes of staff working with older people experiencing mental health issues ${ }^{12}$ highlighted the following: a lack of staff training in dementia, crisis work taking longer to manage, and pressure on resources. However, $\mathrm{McNab}$ et al investigated on older people's home treatment teams (HTTs) that provided support to carers and signposted the person with dementia to local services and reported high patient satisfaction and a reduction in bed occupancy. ${ }^{13}$ There is, however, a systematic review of crisis teams that identified only lowlevel quality evidence for the effectiveness of such teams in reducing admissions to hospital, ${ }^{14}$ suggesting a gap in the current literature. Potentially, a separate service for older adults is necessary as there is an increased likelihood that ill health impacting mental health requires specialized care.

Overall, the evidence surrounding older people's services that work with people who have dementia and are experiencing a crisis is dated, and it is unclear if anything has changed. This literature review intends to identify studies specifically targeting older people with dementia who experience a crisis to provide an update and highlight where future research is required. The online survey allows for an update of current knowledge on services that are working with people with dementia in crises.

\section{Aims}

- To conduct a systematic review to investigate the impact of crisis teams on outcomes, such as reducing hospital admissions for people with dementia, in comparison with usual care.

- Use an online scoping survey of teams managing crisis in people with dementia in England to broaden our understanding of what is currently working in practice.

\section{Methods}

\section{Systematic review}

A previous systematic review on the effectiveness of older adult crisis teams was carried out for the Home Treatment Programme study as part of the Support at Home Interventions to Enhance Life in Dementia project (RP-PG-06061083) led by Professor Orrell. The grant for the Achieving Quality and Effectiveness in Dementia Using Crisis Teams (AQUEDUCT) study was awarded to Professor Orrell and is developed from the Home Treatment Programme study. So, the updated systematic review is built on the original work carried out, ${ }^{14}$ whereas the scoping survey aims to fill the gap in the existing literature by demonstrating the current use of older adult crisis teams in England.

\section{Types of articles included in the review}

All methodological designs were eligible for inclusion in this review, such as controlled comparison studies, including randomized controlled trials (RCTs), controlled before and after studies, interrupted time series, observational studies, theoretical papers, and government frameworks and policies. Studies were included if a crisis experienced by a person with dementia met the criteria of "an urgent need for an assessment and intervention for a person living in the community". ${ }^{15}$

\section{Types of comparison groups}

Experimental intervention: Older people with dementia in receipt of any mental health crisis resolution/home treatment intervention.

Control: Control groups included "treatment as usual," standard community treatment, waiting list controls, and matched controls.

\section{Types of outcome measures}

Primary outcomes included the number of hospital admissions, length of hospital stay, maintenance of community residence, and patient quality of life. Secondary outcome measures included the patient's cognition, activities of daily living, mortality rates, use of medication, level of patient 
and/or carer satisfaction, level of service use, and health and social care costs.

\section{Types of participants}

Participant inclusion criteria for the studies were participants aged 65 years or older, with a diagnosis of dementia, and living in the community.

\section{Search methods for identification of studies}

Electronic searches of databases searched on July 27, 2015 included MEDLINE, EMBASE, PsycINFO, CINAHL, and LILACS, and gray literature sources were also included. A previous systematic search identified studies dating back to 1965-2008, and consequently this search period ranged from January 2008 to July 27, 2015. ${ }^{14}$ The search terms used for database searches included old*, elder*, aged, patient care management, patient care team, case management, intensive case management, care management, managed care programs, community mental health team, specialist mental health service, community mental health, community mental health services, community mental health centers, community care, long-term care, community based long-term care, dementia care, intermediate care, crisis resolution, crisis intervention, home treatment, home care, home nursing, home care services, care coordination, care pathway, managed care, outreach, assertive outreach, disease management, carer support, family intervention, admiral nursing, assessment and service arrangement, health services for the aged, geriatric health service, and family-based therapy. The search terms were identified in a previous systematic review, ${ }^{14}$ and the review was updated. A large number of search terms were used to be as inclusive as possible of potentially relevant work being undertaken in practice.

\section{Data collection and analysis}

In accordance with the defined inclusion criteria, titles, and abstracts of citations obtained from the search were examined by a researcher (AS) and irrelevant articles discarded. For the citations considered potentially relevant the full text was obtained and further information was sought from study authors if required. Two independent reviewers (AS, JY) assessed the methodological quality of papers using the Critical Appraisal Skills Programme Centre (CASP) checklists for cohort and case-control studies. ${ }^{16}$ All studies were assigned a level of evidence of low, acceptable, or high according to the criteria included in the checklists. Where there was difference of opinion, the two reviewers discussed the study using the checklist until an agreement was reached.

\section{Online scoping survey}

\section{Service identification procedure}

The online survey was developed drawing on the Memory Clinics Audit 2014 template. The NHS England website was used to identify all appropriate NHS Trusts, and these were entered onto a Microsoft Excel spreadsheet. Internet searches were carried out to determine if each Trust had a specific dementia crisis service, and where contact details were available, a follow-up telephone call to the service was made to confirm the manager's contact details. There were, however, many Trusts where the existence of specific dementia crisis services was unclear. In these instances, each Trust website was accessed to identify contact details for the Community Mental Health Team (CMHT) and/or memory clinic. When contact was made, it was explained that contact details were being sought for a crisis service working with people with dementia, with the intention of disseminating a survey to all managers of these services across England. When the older adult or adult service was contacted, the researcher clarified that the demographic of patients included people with dementia. The name of the service lead or manager and email address were collected for each service within every Mental Health Trust in England. Wherever possible, the contact person within the service was also asked to identify other services within their Trust that worked in a similar capacity, and any other contacts were followed up. The AQUEDUCT (RP-PG-0612-20004) programme manager used her direct work email to contact each service manager to encourage completion and to provide a point of reference for the survey.

\section{Survey design}

Information was provided prior to entering the survey, including an introduction to the AQUEDUCT study, the purpose of the survey, definition of a crisis, and how the results would inform the research study. The survey included 29 questions and was conducted using SurveyMonkey software; it was designed to be completed by the manager of the service. Screening questions included the type of service that the manager was responsible for and whether they provided specialist interventions for people with dementia and their carers in the community. If the respondent stated that crisis was not within the scope of their service, they were automatically exited from the survey. 
The online scoping survey collected responses on what type of service the respondent was responsible for, whether it was a specialist service for older people in crisis, employer type, job role, work grade, and years in practice. In terms of organizational details, questions related to days and hours of operation, composition of team, service eligibility criteria, referral process, primary diagnosis and primary needs of those entering the service, and time spent in profession specific versus generic working. In relation to referrals, this included the average number of referrals per week, average number of service users on a person's caseload, whether the service follows a care pathway, interventions and assessments used, and the challenges and benefits to delivering home treatment. The respondents were also asked if their service participated in research and whether they would like to be contacted in the future.

\section{Ethics}

Ethical approval was not required for the scoping survey as the work carried out was not considered to be a research study. All questions required for the survey were sent to Research and Innovation, Nottinghamshire Healthcare NHS Foundation Trust to determine the suitability of questions asked. The scoping survey was approved by the sponsor Trust, with staff member informed consent deemed unnecessary as individuals were not directly interviewed and identifiable information was not collected, unless the person completing the survey volunteered his/her contact details after having completed the survey.

\section{Results}

\section{Systematic review} Included studies

A total of 5,344 references were identified in the initial search of the databases; after duplicates were removed $(n=1,759)$, 3,509 were excluded by screening of title and abstract only. A further 71 papers were excluded on the basis that either 1) they did not include people with dementia or 2) they did not include working with people with dementia experiencing crisis. Three papers from this search and a further four studies identified in a previous search were included in this review (Figure 1). ${ }^{14}$ These comprised six cohort studies, ${ }^{17-22}$ and a non-randomized concurrent control treatment outcome trial. ${ }^{23}$ Table 1 summarizes the key points of each study.

\section{Quality assessment}

An overall CASP assessment of each study considered whether the evidence was high quality $(++)$, acceptable $(+)$, or low ( 0 ) and is reported in Table 2 . One study provides high

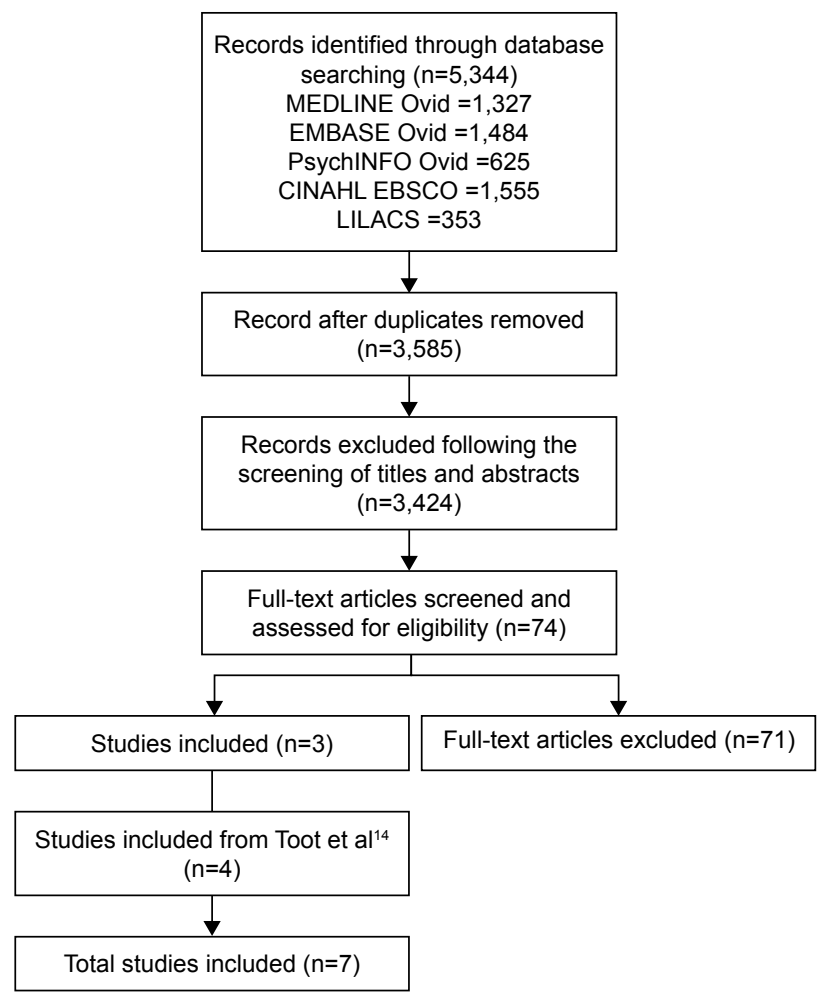

Figure I Consort diagram of included studies.

methodological quality, ${ }^{18}$ four studies provide acceptable methodological quality, ${ }^{17,20,22,23}$ and two studies were considered to have low methodological quality. ${ }^{19,21}$

\section{Studies comparing hospital admissions for people with dementia with or without access to a crisis service}

Villars et $\mathrm{al}^{22}$ reviewed a geriatric team in Toulouse providing an individualized care plan for people experiencing behavioral and psychological symptoms of dementia (BPSD) based on observations during the person's hospital stay, through telephone support. The main outcome included early emergency room rehospitalization. The results suggested a reduced length of stay over 2 years but this difference was not significant $(p=0.56$ ) for those in receipt of telephone support compared to the previous year.

Johnson et $\mathrm{a}^{23}$ engaged a specialist multidisciplinary team to provide support to people with dementia experiencing psychiatric complications with the intention of reducing psychiatric hospitalization in Kansas. The results were compared to a control group of participants previously hospitalized in an inpatient psychiatric hospital. For those in receipt of the intervention, there was a decrease in mortality rates and significant decrease in rehospitalization with people remaining in their homes for longer and significant improvements in caregiver outcomes $(p \leq 0.001)$ and 


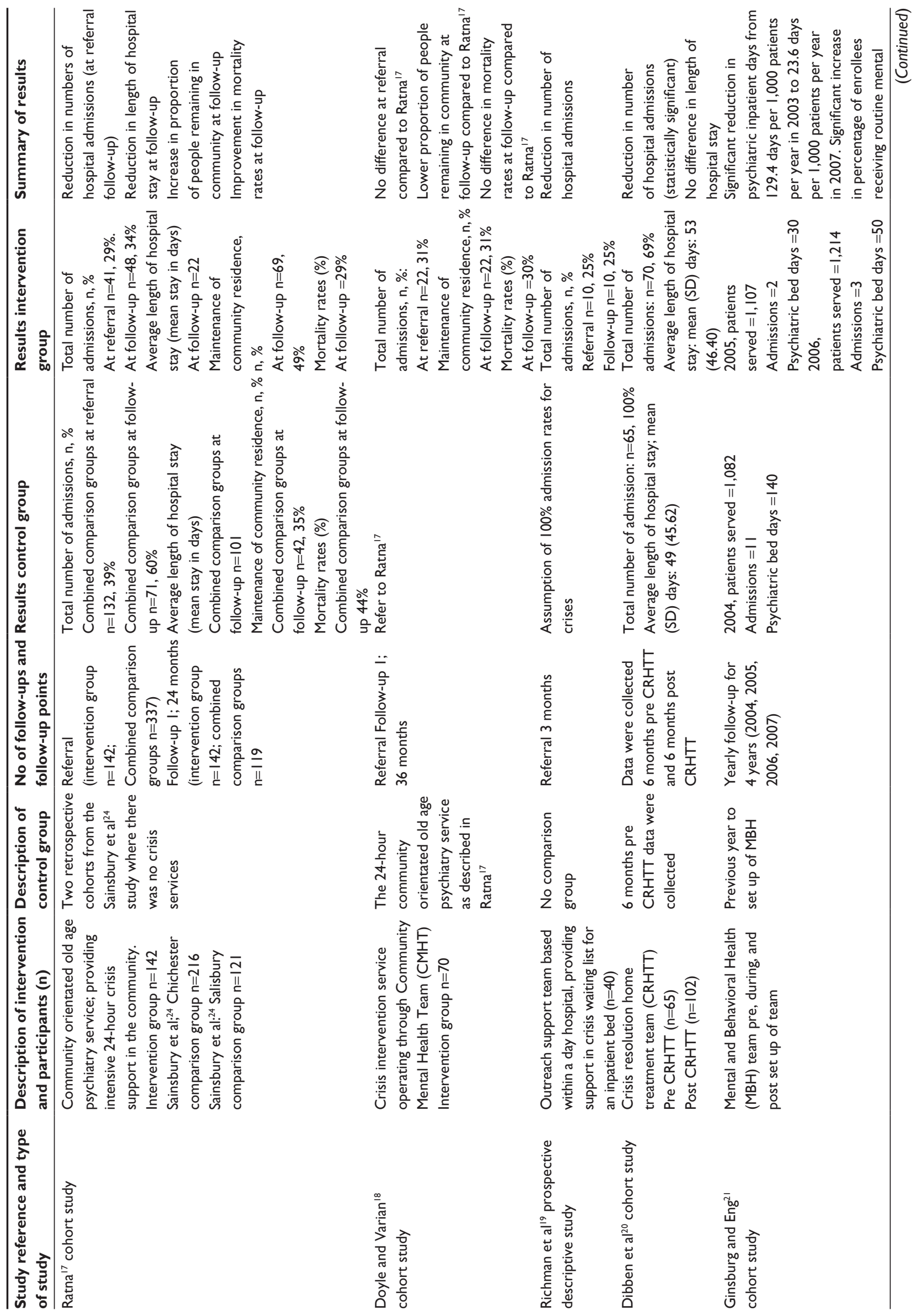




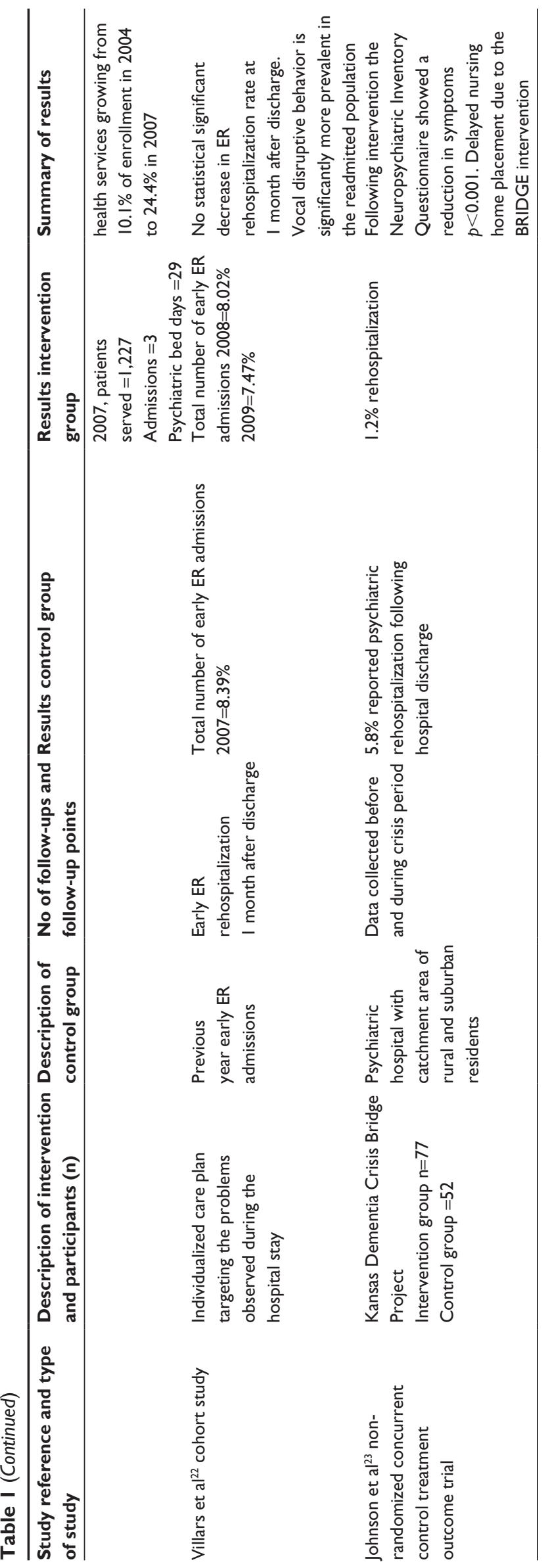

caregiver-reported neuropsychiatric symptoms in people with dementia $(p \leq 0.01)$.

Ginsburg and Eng ${ }^{21}$ reviewed a new Mental and Behavioural Health Team for older people with dementia or mental illness in the community compared to the previous year when there was no team in San Francisco. The primary outcomes include number of patients seen, psychiatric admissions, and bed days. The study demonstrated a reduction in psychiatric inpatient days compared to previous years.

Dibben et $\mathrm{al}^{20}$ examined the effectiveness of a Crisis Resolution HTT service including working with older adults with a functional or organic diagnosis in West Suffolk. The service was extended to include older people due to the closure of a dementia ward and 2-day hospitals. The study found a significant reduction in hospital admissions postsetup of the crisis resolution HTT ( $p \leq 0.001$ ), but there were no significant changes in the other outcomes of bed days and level of satisfaction of service user.

Richman et al ${ }^{19}$ conducted a naturalistic evaluation of a community outreach support team for older adults with mental illness in crisis in Cheshire and Wirral Partnership NHS Trust. The team was introduced in response to the closure of 20 beds in the geriatric ward, aiming to reduce hospital admissions and bed occupancy, encourage early discharge, and support patients at home. Thirty participants were supported in the community and 10 participants were admitted to hospital over the timeframe of the study, suggesting that this type of service might be beneficial in reducing the number of hospital admissions.

Doyle and Varian ${ }^{18}$ compared a 24-hour crisis service offered in Folkstone, Kent with a 9:00 to 17:00 crisis intervention team (data from Ratna ${ }^{17}$ ) for older people with mental health problems, including dementia. The main outcomes included number of psychiatric hospital admissions, maintenance of community residence, and mortality rates. There was a greater referral rate in the 24-hour service $(p \leq 0.01)$, a lower proportion of people remaining in the community in the 24-hour service at follow-up compared to the control group $(p \leq 0.001)$, and no difference in mortality rates.

Ratna ${ }^{17}$ provided a community-based psychogeriatric service in North London for older people with mental illness, including dementia. The main outcomes included number of psychiatric admissions, length of hospital stay, maintenance of community residence, and mortality rates. A previously conducted study provided their control group data. ${ }^{24}$ Ratna $^{17}$ demonstrated a reduction in number of hospital admissions and bed days, an increase in proportion of community residence, and decreased mortality rates. 


\section{Online scoping survey Demographics}

Two hundred and thirty-four individual services potentially managing crises in people with dementia were identified across England, 200 of which had available contact details for managers or service leads who ran the service or ran a number of services. Sixty-two managers, representing 23 English counties, began the online questionnaire, although two managers did not provide consent at the start of the survey and were automatically exited and three people voluntarily exited. The survey was completed in full by 22 respondents $(35 \%)$, and the median number of questions answered by respondents was 13 (out of 29).

The names of services varied and included Dementia and Intensive Support Team, Mental Health Service for Older People, Memory Assessment Service (MAS), Mental Health Intensive Recovery, Dementia Crisis Support Team, Dementia Rapid Response Team, and Intensive Recovery Intervention Service. Overall, 49 of the respondents (86\%) considered their service to provide a specialist intervention/support for people with dementia and their carer experiencing an acute crisis at home. Twenty-seven respondents listed their managerial role as responsible for a CMHT (or similar) (47.4\%), 24 as responsible for a HTT (or similar) (42.1\%), and six as responsible for a memory service (10.5\%). Table 3 and Figures 2 and 3 summarize the characteristics of these three models.

\section{MASs}

Of the six teams who identified themselves as a MAS, four teams indicated their employers (three NHS and one local authority). All teams operated Monday-Friday between 9:00 and 17:00, all used eligibility criteria and had a screening process, and only one team used a standardized care pathway or protocol.

The median number of referrals received by memory assessment teams per week was 18 , ranging from six to 20 . The median percentage of referrals with a primary diagnosis of dementia across teams was $85 \%$ ranging from $20 \%$ to $100 \%$. Teams received referrals from general practitioners (GPs), consultant psychiatrists, outpatients, acute mental health, acute physical health, liaison psychiatry, other health or social care, self-referral, and carer referral. Team leaders were nurses with the exception of one who was an occupational therapist (Figure 3 for team composition), and on an average team members spent $80 \%$ of their time carrying out profession-specific work.

The highest ranked primary care needs of patients referred to the services were behavioral and psychological (eg, anxiety or low mood, delusions, hostility or aggression, and wandering) and the lowest ranked were environmental factors (eg, physical hazards around the home, unable to access essential amenities). The highest ranked intervention utilized by teams was specialist, professional health care practitioner input (eg, medication review, occupational therapy assessment, and clinical psychology input), and the lowest ranked intervention was home care support (eg, provision of home care services).

\section{$\mathrm{CMHTs}$}

Of the 27 teams who identified themselves as a CMHT, 19 teams indicated their employers were the NHS. Sixteen teams indicated their operational hours, 15 of which operated Monday-Friday 9:00-17:00, with one team operating Monday-Sunday and offering an extended hours service. Seven teams used eligibility criteria and three teams stated that they did not. Ten teams indicated that they had a screening process, with four teams using a care pathway/protocol and five teams indicating that they did not use a care pathway or protocol. The median number of referrals received by CMHTs per week was 10, ranging from zero to 25 .

The median percentage of referrals with a primary diagnosis of dementia across teams was $72.5 \%$, ranging from $20 \%$ to $100 \%$. Teams received referrals from GPs, consultant psychiatrists, outpatients, acute mental health, acute physical health, liaison psychiatry, community mental health recovery/community recovery teams, HTTs, the voluntary sector, other health or social care, self-referral, carer referral, primary care liaison teams, and memory services; one indicated that they operated an open referral system. Team leaders included mental health practitioners, nurses, occupational therapists, psychologists, and social workers (see Figure 3 for more details and team composition), and on an average team members spent $40 \%$ of their time carrying out profession-specific work.

The highest ranked primary needs of patients referred to the services were environmental factors and the lowest ranked were behavioral and psychological factors. The highest ranked intervention utilized by teams was environmental (eg, equipment, communication devices, and assistive technology) and the lowest ranked was specialist, professional health care practitioner input.

\section{HTTs}

Of the 24 teams who identified themselves as HTT, 21 indicated their employer type (20 NHS, 1 social enterprise). Twenty teams stated their operational hours, one of which operated Monday-Friday 9:00-17:00. While 19 teams operated Monday-Sunday, 17 of these offered an extended hours service, with two offering a 24-hour service. Out of the 17 teams who responded, 16 of these specified they had 
Table 2 Quality assessment of included studies

\begin{tabular}{|c|c|c|c|c|c|c|c|c|}
\hline Study & $\begin{array}{l}\text { I. Did } \\
\text { the study } \\
\text { address } \\
\text { a clearly } \\
\text { focused } \\
\text { issue? }\end{array}$ & $\begin{array}{l}\text { 2. Was } \\
\text { the cohort } \\
\text { recruited } \\
\text { in an } \\
\text { acceptable } \\
\text { way? }\end{array}$ & $\begin{array}{l}\text { 3. Was } \\
\text { exposure } \\
\text { accurately } \\
\text { measured } \\
\text { to minimize } \\
\text { bias? }\end{array}$ & $\begin{array}{l}\text { 4. Was the } \\
\text { outcome } \\
\text { accurately } \\
\text { measured } \\
\text { to minimize } \\
\text { bias? }\end{array}$ & $\begin{array}{l}\text { 5a. Have authors } \\
\text { identified all } \\
\text { important } \\
\text { confounds? }\end{array}$ & $\begin{array}{l}\text { 5b. Have } \\
\text { they taken } \\
\text { account on } \\
\text { the confounds } \\
\text { in the design/ } \\
\text { analysis? }\end{array}$ & $\begin{array}{l}\text { 6a. Was the } \\
\text { follow-up } \\
\text { of subjects } \\
\text { complete? }\end{array}$ & $\begin{array}{l}\text { 6b. Was the } \\
\text { follow-up } \\
\text { of subjects } \\
\text { long } \\
\text { enough? }\end{array}$ \\
\hline \\
\hline Dibben & Yes & Yes & Yes & Yes & Yes & Yes & Yes & Yes \\
\hline
\end{tabular}

et al ${ }^{20}$

\begin{tabular}{|c|c|c|c|c|c|c|c|}
\hline $\begin{array}{l}\text { Ginsburg } \\
\text { and Eng }\end{array}$ & Yes & Yes & Yes & Yes & $\begin{array}{l}\text { No mention of } \\
\text { what common } \\
\text { mental disorders are } \\
\text { experienced } \\
\text { Number of ppts with } \\
\text { dementia not reported }\end{array}$ & No & Yes \\
\hline $\begin{array}{l}\text { Doyle } \\
\text { and }\end{array}$ & Yes & Yes & Yes & Yes & Yes & Yes & Yes \\
\hline
\end{tabular}

$\operatorname{Varian}^{18}$

\begin{tabular}{|c|c|c|c|c|c|c|c|c|c|}
\hline $\begin{array}{l}\text { Richman } \\
\text { et al }{ }^{19}\end{array}$ & Yes & Yes & Yes & Yes & \multicolumn{2}{|c|}{$\begin{array}{l}\text { No control group } \\
\text { identified }\end{array}$} & No control group & Yes & Yes \\
\hline Ratna $^{17}$ & Yes & Yes & Yes & Not known & Yes & & Yes & Yes & Yes \\
\hline $\begin{array}{l}\text { Villars } \\
\text { et } \mathrm{al}^{22}\end{array}$ & Yes & Yes & Yes & Yes & Yes & & \multicolumn{2}{|c|}{$\begin{array}{l}\text { Analyses have Yes } \\
\text { not differentiated } \\
\text { between severe } \\
\text { and mild dementia }\end{array}$} & No \\
\hline Study & $\begin{array}{l}\text { I. D } \\
\text { stud } \\
\text { a cle } \\
\text { focu }\end{array}$ & ress & $\begin{array}{l}\text { 2. Did the authors } \\
\text { use an appropriate } \\
\text { method to answer } \\
\text { their question? }\end{array}$ & \multicolumn{2}{|c|}{$\begin{array}{l}\text { 3. Were the } \\
\text { cases recruited } \\
\text { in an acceptable } \\
\text { way? }\end{array}$} & $\begin{array}{l}\text { 4. Were the } \\
\text { controls } \\
\text { selected in an } \\
\text { acceptable way? }\end{array}$ & \multicolumn{2}{|c|}{$\begin{array}{l}\text { 5. Was exposure } \\
\text { accurately } \\
\text { measured to } \\
\text { minimize bias? }\end{array}$} & $\begin{array}{l}\text { 6a. What } \\
\text { confounding factors } \\
\text { have the authors } \\
\text { accounted for? }\end{array}$ \\
\hline \multicolumn{10}{|c|}{ CASP checklist case-control studies: } \\
\hline $\begin{array}{l}\text { Johnson } \\
\text { et } \mathrm{al}^{23}\end{array}$ & Yes & & Yes & Yes & & Yes & Yes & & $\begin{array}{l}\text { Differences between } \\
\text { control and } \\
\text { intervention groups } \\
\text { No reporting of } \\
\text { participants family } \\
\text { situation }\end{array}$ \\
\hline
\end{tabular}

Abbreviations: CASP, Critical Appraisal Skills Programme Centre; CRHTT, crisis resolution home treatment team; CMHT, community mental health team; ER, emergency room; NP, neuropsychiatric; ppts, patients. 


\begin{tabular}{|c|c|c|c|c|c|c|}
\hline 7. What are the results of this study? & $\begin{array}{l}\text { 8. How } \\
\text { precise } \\
\text { are the } \\
\text { results? }\end{array}$ & $\begin{array}{l}\text { 9. Do you } \\
\text { believe } \\
\text { the } \\
\text { results? }\end{array}$ & $\begin{array}{l}\text { 10. Can the } \\
\text { results be } \\
\text { applied to } \\
\text { the local } \\
\text { population? }\end{array}$ & $\begin{array}{l}\text { II. Do the } \\
\text { results of } \\
\text { this study fit } \\
\text { with other } \\
\text { available } \\
\text { evidence? }\end{array}$ & $\begin{array}{l}\text { 12. What are the } \\
\text { implications of this study } \\
\text { for practice? }\end{array}$ & Rating \\
\hline
\end{tabular}

Admissions reduced by $31 \%$

General trend for greater satisfaction in

carers and service users

No difference in involuntary admissions

No odds ratios

Increased access to mental health services

Reduction in admissions and psychiatric

bed days

High staff satisfaction with treatment

Patients in long stay hospital beds similar for both groups

Both services good at predicting when hospitalization needed and mobilizing support to prevent further admissions More people in residential care in office hours group - although more residential care also available in this group 30 admissions to inpatient psychiatric care were avoided through the establishment of this team.

The population seen in crisis was similar to that referred to other services

Assessments made in the home are as effective in determining who should go to hospital and who can be managed in the community

This model is effective at stabilizing patients to enable care in the community No significant differences in early ER rehospitalization

$\begin{array}{ll}\text { Quite Yes } \quad \text { Yes within } & \text { Yes } \\ & \text { reason - all health } \\ & \text { care contexts are } \\ & \text { different }\end{array}$

Not Yes Potentially, in

very supported living environments

Good Yes

Yes

Not a lot of other evidence is discussed in relation to the findings

Yes

Yes in areas where Yes CMHT exists but domiciliary crisis services do not Good Yes Yes - but few areas would be able to support a 24-hour crisis service

Quite Yes Yes Yes

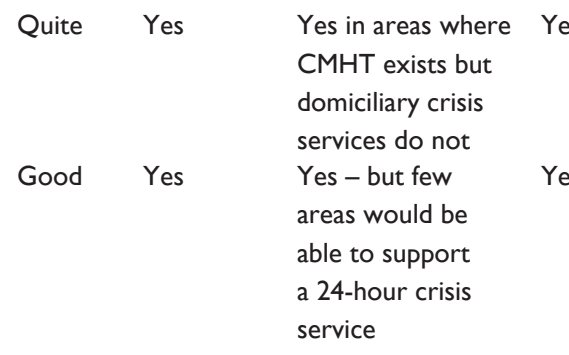

Yes
Recommendation of use of $\quad+$ CRHTT for older people

Mental health professionals should be a part of integrated living teams

Crisis teams operating within office hours can be as effective as 24 -hour teams

0

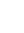

This kind of intervention

0 may reduce admission to inpatient psychiatric care Crisis services are able to + support people at home

\begin{tabular}{|c|c|c|c|c|c|c|}
\hline $\begin{array}{l}\text { 6b. Have the authors } \\
\text { taken account of the } \\
\text { potential confounds } \\
\text { in design/analysis? }\end{array}$ & $\begin{array}{l}\text { 7. What are } \\
\text { the results of } \\
\text { this study? }\end{array}$ & $\begin{array}{l}\text { 8. How } \\
\text { precise } \\
\text { are the } \\
\text { results? }\end{array}$ & $\begin{array}{l}\text { 9. Do you } \\
\text { believe the } \\
\text { results? }\end{array}$ & $\begin{array}{l}\text { 10. Can the } \\
\text { results be } \\
\text { applied to the } \\
\text { local population? }\end{array}$ & $\begin{array}{l}\text { II. Do the results } \\
\text { of this study } \\
\text { fit with other } \\
\text { available evidence? }\end{array}$ & Rating \\
\hline $\begin{array}{l}\text { No statistical } \\
\text { adjustment }\end{array}$ & $\begin{array}{l}\text { Reduction in } \\
\text { NP symptoms, } \\
79 \% \text { resolution in } \\
\text { crisis, less hospital } \\
\text { admissions than } \\
\text { control group, } \\
\text { delayed nursing } \\
\text { home placement }\end{array}$ & $\begin{array}{l}\text { Quite - no } \\
\text { confidence } \\
\text { intervals and } \\
\text { no reporting } \\
\text { of ppts who } \\
\text { declined }\end{array}$ & Yes & $\begin{array}{l}\text { Can be applied } \\
\text { in areas where } \\
\text { complete lack of } \\
\text { services. Not sure } \\
\text { how well these } \\
\text { findings integrate } \\
\text { into UK health } \\
\text { system }\end{array}$ & Yes & + \\
\hline
\end{tabular}


Table 3 Characteristics of survey respondents according to team model

\begin{tabular}{|c|c|c|c|c|}
\hline Characteristic & $\begin{array}{l}\text { Memory assessment } \\
\text { service (\%) }\end{array}$ & $\begin{array}{l}\text { Community mental } \\
\text { health team (\%) }\end{array}$ & $\begin{array}{l}\text { Home treatment } \\
\text { team (\%) }\end{array}$ & $\begin{array}{l}\text { Total responses for } \\
\text { each survey question }\end{array}$ \\
\hline \multicolumn{5}{|l|}{ Employer type } \\
\hline $\mathrm{NHS}$ & $3(75)$ & $19(100)$ & $20(95)$ & 44 \\
\hline Local authority & I (25) & 0 & 0 & \\
\hline Social enterprise & 0 & 0 & I (5) & \\
\hline \multicolumn{5}{|l|}{ Days of operation } \\
\hline Monday-Friday & $3(100)$ & $15(94)$ & I (5) & 39 \\
\hline Monday-Sunday & 0 & I (6) & $19(95)$ & \\
\hline \multicolumn{5}{|l|}{ Hours of operation } \\
\hline $9: 00-17: 00$ & $3(100)$ & $15(94)$ & I (5) & 39 \\
\hline Extended eg, 7:00-22:00 & 0 & I (6) & $17(85)$ & \\
\hline 24 hours & 0 & 0 & $2(10)$ & \\
\hline \multicolumn{5}{|l|}{ Eligibility criteria } \\
\hline Yes & $3(100)$ & $7(70)$ & $16(94)$ & 30 \\
\hline No & 0 & $3(30)$ & I (6) & \\
\hline \multicolumn{5}{|l|}{ Referral/screening process } \\
\hline Yes & $3(100)$ & $10(100)$ & $16(94)$ & 30 \\
\hline No & 0 & 0 & I (6) & \\
\hline \multicolumn{5}{|l|}{ Pathway/protocol } \\
\hline Yes & I (33) & $4(44)$ & $9(60)$ & 27 \\
\hline No & $2(67)$ & $5(56)$ & $6(40)$ & \\
\hline
\end{tabular}

eligibility criteria for their service. Sixteen teams indicated that they had a screening process and one did not. Nine teams used a care pathway/protocol and six teams indicated that they did not use any form of care pathway or protocol.
The median number of referrals received by memory assessment teams per week was 10, ranging from zero to 50 . The median percentage of referrals with a primary diagnosis of dementia across teams was $60 \%$, ranging from

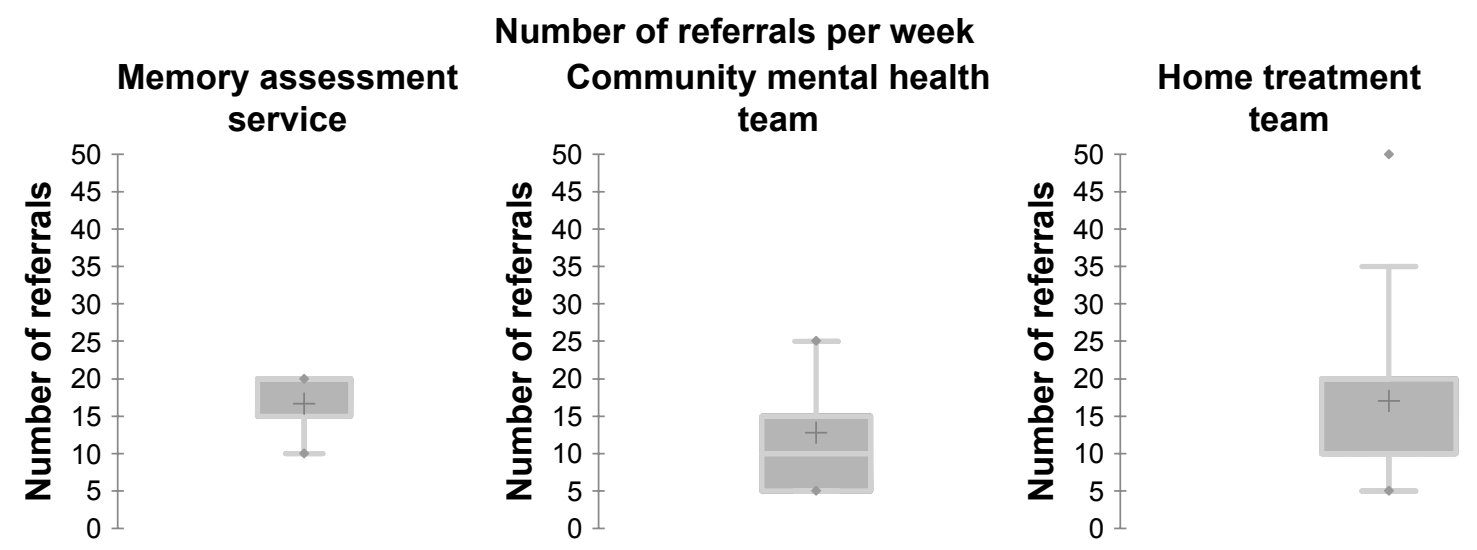

Percentage of patients with primary diagnosis of dementia
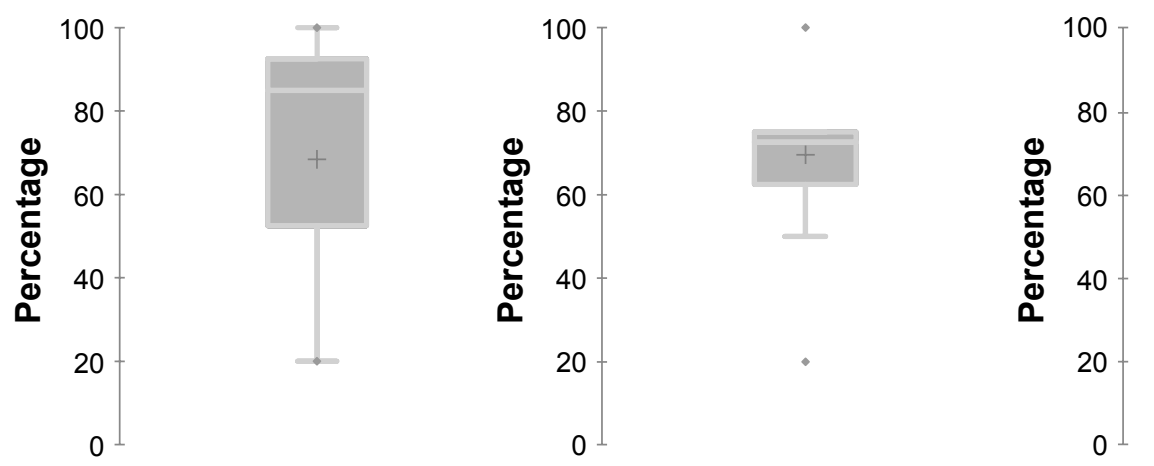

Figure 2 Referral characteristics for each model of team managing crisis in dementia. 


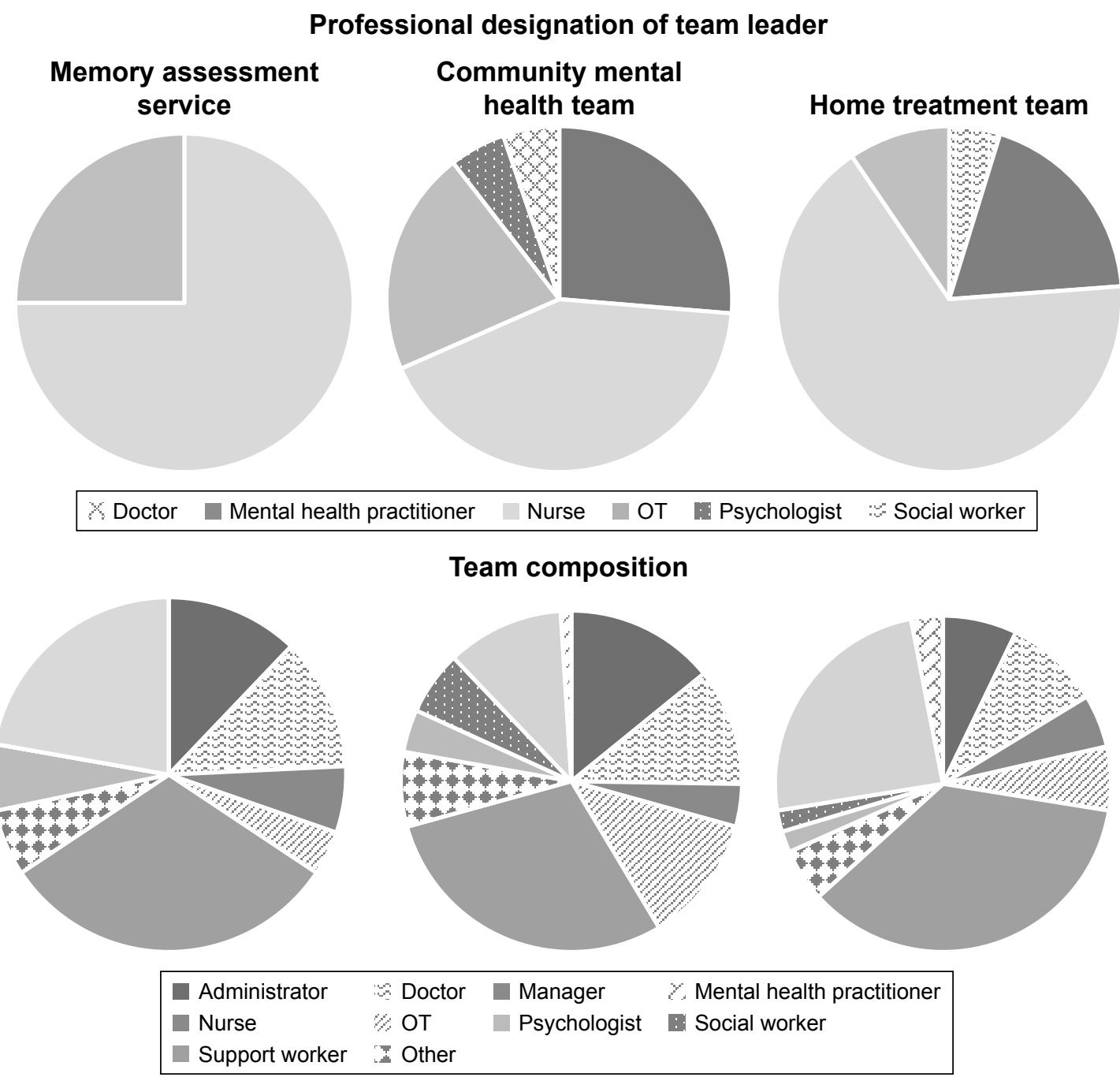

\section{Percentage of time spent doing profession-specific work}
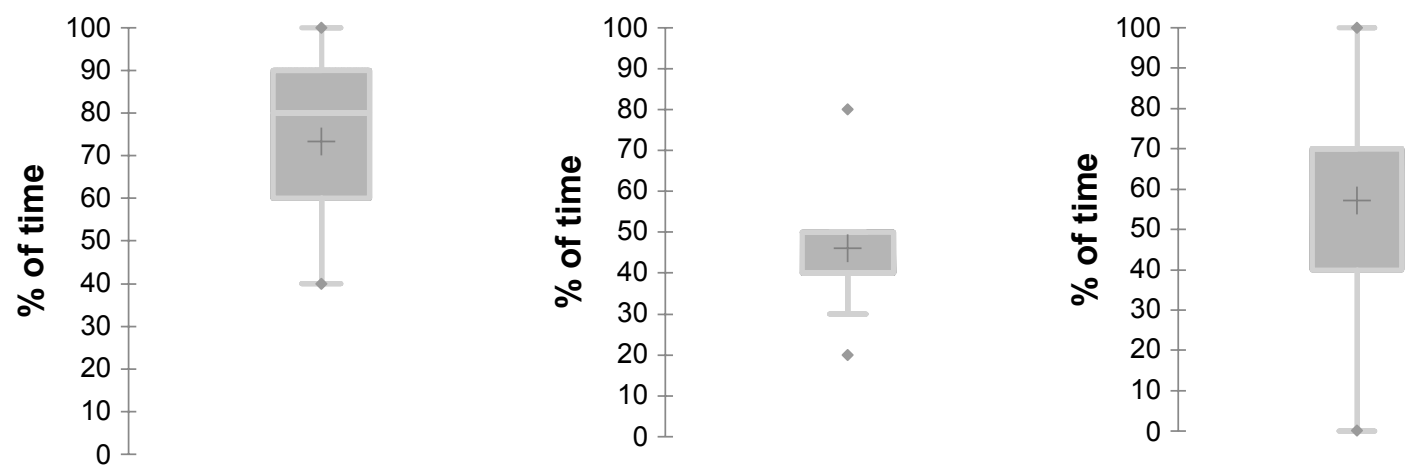

Figure 3 Characteristics of teams managing crises in people with dementia.

$30 \%$ to $100 \%$. Teams received referrals from GPs, consultant psychiatrists, outpatients, acute mental health, acute physical health, liaison psychiatry, community mental health recovery/community recovery teams, HTTs, the voluntary sector, other health or social care, self-referral, carer referral, ambulance services, and single point of access; one indicated that they operated an open referral system. Team leaders included doctors, mental health practitioners, nurses, and occupational therapists (see Figure 3 for more details and team composition), and on an average team members spent $70 \%$ of their time carrying out professionspecific work. 
The highest ranked primary care needs of patients referred to the services were behavioral and psychological factors and the lowest ranked were environmental factors. The highest ranked intervention utilized by teams was specialist, professional health care practitioner input and the lowest ranked was environmental.

\section{Assessment measures}

Across the three team models, there were no differences in the standardized assessments used and all models used a range of different assessments. Twenty-six respondents (96.2\%) use standardized assessments, and 24 different assessment tools were listed. The measures tended to be cognitive assessment tools, such as the Addenbrooke's Cognitive Examination-III, the Montreal Cognitive Assessment, and the Mini Mental State Examination. Most teams also used an assessment for the presence of anxiety or depression such as the Hospital Anxiety and Depression Scale or the Cornell Scale for Depression in Dementia. Notably, risk assessment was only mentioned by one team and no measures of crisis, quality of life, or caregiver burden were listed.

\section{Challenges and benefits of delivering home treatment interventions}

The respondents identified a number of challenges to delivering home treatment interventions. Examples include recognizing the gap between health and social care systems, the desire to have a social worker working within the team, timely nature to accessing social care, lack of access to community services, low staffing levels and high workloads, difficulty associated with the complexity of cases, and the geographical spread of the service. Examples of the benefits of delivering a home treatment include remaining patient centered, improved quality of life for the patient, supporting service users to remain in their own home for longer, avoidance of unnecessary hospital admissions, and the opportunity to provide intensive support to the people with dementia and their carer that could prevent future crises.

\section{Discussion}

National policies support the use of similar services for older people with mental health problems, ${ }^{25-27}$ and our survey, despite being limited in its response rate, contributes to our current understanding of crisis teams working with people with dementia in practice.

There was, however, a satisfactory initial uptake rate (33.5\%) and a good completion rate $(35 \%)$. This appears to be a similar response rate to a previously reported review of email responses to surveys. ${ }^{28}$ The responses indicate that the provision of services is inconsistent and practice delivery varies greatly. This variation includes the naming of services, setup and delivery, policies, and procedures; however, this aligns with previous research. ${ }^{10,14}$ This may be a contributing factor to the lack of rigorous evidence and evaluation of these types of services in the literature. ${ }^{14}$

The review has identified more studies than found in the previous systematic review of the literature, ${ }^{14}$ which has furthered our knowledge and understanding of crisis teams. Regarding the systematic review in accordance with the Oxford Centre for Evidence-based Medicine used in the previous systematic review, ${ }^{14}$ two of the three newly included papers were of grade C. ${ }^{21,22}$ There was one paper, however, ${ }^{23}$ that was considered grade $\mathrm{B}$, demonstrating a slight improvement in the reported study design. It is important to note, however, that it was only possible to report on the availability of evidence generated from the search and there may have been reports of service delivery in practice that were subsequently missed. However, this search of the literature did not identify high-quality studies such as RCTs, and predominantly cohort studies were included that compared findings to previous years of running the service or to a comparison group from a previously conducted study. In one reported study, the control group was taken from a study published in 1965, and arguably this is too dated as services have changed since this time. The majority of studies used a mixed sample of older people with dementia or mental illness, ${ }^{17-21}$ or working age and older age, but only provided analysis of overall results. Also, the reporting of effect sizes for included studies was attempted but not always available from the original paper. Consequently, due to the lack of quality in study design and reporting, it was not possible to synthesize the results across included studies in a meaningful way. In addition, the wide geographical spread makes it difficult to draw assumptions due to the heterogeneity of the included studies.

Most studies provided adequate $(+)$ methodological quality, and there is some evidence for crisis services for older people with mental health issues positively impacting on reducing the number of hospital admissions, ${ }^{17,19,20}$ readmissions, ${ }^{23}$ length of stay, ${ }^{21,22}$ and mortality rates. ${ }^{17,23}$ This does, however, need to be interpreted with caution due to the small number of studies, variable study designs, and lack of statistical rigor. Consequently, the systematic review was limited by a lack of good quality studies, leading to lower quality evidence. The literature review demonstrated no significant improvement in the design or reported clinical 
effectiveness of studies evaluating crisis team working for people with dementia.

When comparing and contrasting the different models of service delivery, operational days and times varied across models with HTTs tending to offer extended hours across 7 days, and some offering a 24/7 service. By contrast, MASs and CMHTs typically provided services Monday-Friday 9:00-17:00, which may partly be due to the evolution of such teams, for example, in response to ward closures where teams and staff members may have been accustomed to working shift patterns. This is also reflective of the overall team composition across all models where nurses feature prominently both as team leaders and as members of staff. However, differences were seen across models in that social workers were included more so in CMHTs, less so in HTTs, and not at all in MASs. However, the role definition of mental health practitioners featured in all teams is unclear and may overlap with other professional disciplines. Further enquiry into the professional boundaries and responsibilities associated with this role is needed.

The percentage of time spent doing profession-specific work differed both within and across models; yet the MASs were reported as doing the largest proportion of professionalspecific work and CMHTs the smallest proportion. Taken into consideration the types of intervention offered by the teams, this is unsurprising as the interventions most frequently used by MASs involved specialist professional health care practitioner input, whereas CMHTs rated environmental interventions as the most frequently used, which may not require profession-specific work to the same extent. MASs and HTTs ranked behavioral needs as the most common type of patient need, whereas CMHTs ranked these as their least common, and in contrast to this they ranked their most common need as environmental, which was the need ranked least common by the MASs and HTTs. This suggests that the three models of crisis services are responding to different patients from diverse circumstances and therefore managing crises differently. It must be acknowledged that all models of service rated family carer factors (eg, burden, physical health, and death of carer) as the second most common type of patient need and also the second most common form of intervention (eg, education, training, and respite), highlighting that despite wide variation there are some elements that straddle all models of care. Much of the research around crisis in older people with dementia considers only BPSD, which could suggest that the work of CMHTs is unrecognized and unresearched due to their focus on environmental and carer-related factors.
Although the median number of referrals per week do not show a large degree of variation across team models, the range suggests that CMHTs and HTTs experience greater variation in the number of referrals. HTTs tended to have more referrals than CMHTs and experienced a greater range of referrals. The MASs and HTTs presented a similar picture of variation in the patients arriving at their service with a primary diagnosis of dementia showing that for some teams in this model all of their patients had a primary diagnosis of dementia, whereas other teams saw as little as $20 \%$ of referrals with a dementia diagnosis. By contrast, CMHTs showed much less variation with most teams seeing approximately $75 \%$ of their patients with a primary diagnosis of dementia. It is surprising to note that, despite receiving referrals from a fewer number of sources, MASs show a large variety in the type of patients accessing the service. Across all three models of service delivery, the majority of teams used eligibility criteria and a screening process and yet still saw patients with a variety of diagnoses and needs. Arguably, the disparate needs of patients referred to services may hinder streamlined service delivery. In conjunction with this, protocols are not routinely used by teams in any model of service delivery. Potentially, this could be because it would be inappropriate to use a protocol with such a wide variety of service users. A specialized dementia protocol, including appropriate clinical measures, coupled with the awareness of when it is appropriate to use such a protocol, would help to facilitate the selection of a suitable intervention for these patients. Additionally, standardized risk assessment or quality of life measures might be more appropriate than cognitive measures to determine change pre-intervention and post-intervention.

Some methodological limitations exist with the scoping survey. In order to invite Managers to participate, correct contact details were essential, yet were often provided over the telephone and required further follow-ups. Furthermore, some Managers, especially those managing across a number of services, might not be able to provide the most detailed picture of the service as they may not interact with the service frequently enough at ground level. The emphasis of the introduction to the survey was on dementia crisis teams, and consequently some Managers may not have felt that the survey was applicable to them if they also worked across other services such as interventions for older people experiencing functional mental health crises. Since services varied greatly, some of the non-completers may have been eligible to participate in the survey. 
The survey, however, was able to identify a variation in the naming and setup of services of teams managing crisis in people with dementia. The survey was designed to enable all teams who manage crisis in people with dementia to participate, whether they were a designated crisis team or a specialist dementia team. This allowed for responses to be gained from teams who may otherwise not have participated and has broadened the understanding of practice in crisis management for people with dementia. Additionally, respondents were able to leave contact details at the end of the survey, which may facilitate recruitment during future stages of the AQUEDUCT research programme. Respondents from a range of areas across England participated, suggesting that responses were gained from a range of teams and were representative of current practice for the country as a whole.

Current research fails to demonstrate full translation of guidelines for crisis resolution teams into practice. ${ }^{29}$ Consequently, future research could look to include gray literature, other methods to assess interventions, qualitative work, and service evaluations. A realist review is needed to unpack the complexities of delivering a complex intervention, identify facilitators and barriers to its applicability across settings, and provide an inclusive perspective of crisis teams working in the United Kingdom. The National Dementia Strategy ${ }^{3}$ aimed to provide good quality care for people living with dementia in the community including responsive crisis services and this can be carried out through the reporting of simple interventions and professional support with the intention of preventing hospital admissions.

\section{Conclusion}

The research evidence for crisis intervention teams for older adults with dementia is predominantly cohort studies. This is problematic in evaluating the effectiveness of the intervention as it is a weaker study design. There is some limited evidence to support the effectiveness of crisis intervention teams for older people with dementia in reducing hospital admissions, but further high-quality evidence is required. The scoping survey revealed a picture of wide variation both within and across the three models of service delivery, and further research is needed to clarify how best to support teams in delivering care for people with dementia who experience a crisis. Clearly defined protocols may be beneficial, particularly when a team's caseload can overlap across functional mental health and dementia, or across ages, in order to clearly define the "best" pathway of care for the person with dementia.

\section{Acknowledgments}

This systematic review and scoping survey were conducted as part of the AQUEDUCT programme funded by the National
Institute for Health Research (NIHR) under its Programme Grants for Applied Research scheme (RP-PG-0612-20004). The AQUEDUCT team acknowledges the support of the National Institute for Health Research Clinical Research Network. The views expressed are those of the authors and not necessarily those of the NHS, the NIHR, or the Department of Health.

\section{Author contributions}

A Streater, DM Coleston-Shields, and M Orrell designed the survey. A Streater, DM Coleston-Shields, J Yates, and M Stanyon analyzed the data. All authors interpreted the data. A Streater carried out the search for the systematic review. A Streater and J Yates carried out the quality appraisal of the included studies. All authors contributed to the drafting of the manuscript and revised and approved the final version.

\section{Disclosure}

The authors report no conflicts of interest in this work.

\section{References}

1. Dementia UK: the full report. Alzheimer's Society. London, UK: Alzheimer's Society; 2007.

2. Alzheimer's Society. Counting the Cost-Caring for People with Dementia on Hospital Wards. London, UK: Alzheimer's Society; 2009.

3. Department of Health. Living Well with Dementia: a National Dementia Strategy. London: Department of Health; 2009.

4. MacNeil Vroomen J, Bosmans JE, van Hout HP, de Rooij SE. Reviewing the definition of crisis in dementia care. BMC Geriatr. 2013; 13:10.

5. Audit Commission. Support for Carers of Older People. London: Audit Commission; 2004.

6. Department of Health. Prime Minister's Challenge on Dementia 2020. London: Department of Health; 2015.

7. Regan C, Cooper C. Crisis resolution teams and older people. In: Johnson S, Needle J, Bindman JP, Thornicroft G, editors. Crisis Resolution and Home Treatment in Mental Health. London, UK: Cambridge University Press; 2008:267-274.

8. Lloyd-Evans B, Bond GR, Ruud T, et al. Development of a measure of model fidelity for mental health Crisis Resolution Teams. $B M C$ Psychiatry. 2016;16(1):427.

9. National Audit Office. Improving Services and Support for People with Dementia. London, UK: National Audit Office; 2007.

10. Cooper C, Regan C, Tandy AR, Johnson S, Livingston G. Acute mental health care for older people by crisis resolution teams in England. Int J Geriatr Psychiatry. 2007;22(3):263-265.

11. Department of Health. Dementia: a State of the Nation Report on Dementia Care and Support in England. London: Department of Health; 2013.

12. Johnson S, Needle J. Introduction. In: Johnson S, Needle J, Bindman J, Thornicroft G, editors. Crisis Resolution and Home Treatment in Mental Health. London, UK: Cambridge University Press; 2008:3-8.

13. McNab L, Smith B, Minardi HA. A new service in the intermediate care of older adults with mental health problems. Nurs Older People. 2006;18(3):22-26.

14. Toot S, Devine M, Orrell M. The effectiveness of crisis resolution/ home treatment teams for older people with mental health problems: a systematic review and scoping exercise. Int J Geriatr Psychiatry. 2011; 26(12):1221-1230. 
15. Hoe J, Ledgerd R, Devine M, Toot S, Challis D, Orrell M. Support at Home: Interventions to Enhance Life in Dementia (SHIELD) Home Treatment Manual 2012 Version 4.

16. Critical Appraisal Skills Programme (CASP). CASP checklists. CASP 2016. Available from: http://www.casp-uk.net/\#!checklists/cb36. Accessed March 13, 2017.

17. Ratna L. Crisis intervention in psychogeriatrics: a two-year follow-up study. Br J Psychiatry. 1982;141:296-301.

18. Doyle H, Varian J. Crisis intervention in psychogeriatrics: a round-theclock commitment? Int J Geriatr Psychiatry. 1994;9(1):65-72.

19. Richman A, Wilson K, Scally L, Edwards P, Wood J. Service innovations. Psychiatrist. 2003;27(9):348-351.

20. Dibben C, Saeed H, Stagias K, Khandaker GM, Rubinsztein JS. Crisis resolution and home treatment teams for older people with mental illness. Psychiatr Bull. 2008;32(7):268-270.

21. Ginsburg IF, Eng C. On-site mental health services for PACE (Program of All-inclusive Care for the Elderly) centers. J Am Med Dir Assoc. 2009;10(4):277-280

22. Villars H, Dupuy C, Soler P, et al. A follow-up intervention in severely demented patients after discharge from a special Alzheimer acute care unit: impact on early emergency room re-hospitalization rate. Int $J$ Geriatr Psychiatry. 2013;28(11):1131-1140.
23. Johnson DK, Niedens M, Wilson JR, Swartzendruber L, Yeager A, Jones K. Treatment outcomes of a crisis intervention program for dementia with severe psychiatric complications: the Kansas bridge project. Gerontologist. 2013;53(1):102-112.

24. Sainsbury P, Costain WR, Grad J. The effects of community service on the referral and admission rates of elderly psychiatric patients. In: World Psychiatric Association Symposium, editor. Psychiatric Disorders in the Aged. 1965:23-37.

25. Department of Health. The Mental Health Policy Implementation Guide. London: Department of Health; 2001.

26. Department of Health. Securing Mental Health for Older Adults. London: Department of Health; 2004.

27. Department of Health. Everybody's Business: Integrated Mental Health Services for Older Adults: a Service Development Guide. London: Department of Health; 2005.

28. Sheehan KB. E-mail survey response rates: a review. J Comput Mediat Commun. 2001;6(2). DOI: 10.1111/j. 1083-6101.2001.tb00117.x.

29. Lloyd-Evans B, Paterson B, Onyett S, et al. National implementation of a mental health service model: a survey of Crisis Resolution Teams in England. Int J Ment Health Nurs. Epub 2017 Jan 11.
Clinical Interventions in Aging

\section{Publish your work in this journal}

Clinical Interventions in Aging is an international, peer-reviewed journal focusing on evidence-based reports on the value or lack thereof of treatments intended to prevent or delay the onset of maladaptive correlates of aging in human beings. This journal is indexed on PubMed Central, MedLine,

\section{Dovepress}

CAS, Scopus and the Elsevier Bibliographic databases. The manuscript management system is completely online and includes a very quick and fair peer-review system, which is all easy to use. Visit http://www.dovepress. $\mathrm{com} /$ testimonials.php to read real quotes from published authors. 